\title{
Some natural zero one laws for ordinals below $\varepsilon_{0}$
}

\author{
Andreas Weiermann and Alan R. Woods \\ 1 Vakgroep Wiskunde, Krijgslaan 281 S22, 9000 Ghent, Belgium \\ Andreas. Weiermann@UGent . be \\ 2 Nedlands W.A 6907 \\ Australia
}

\begin{abstract}
We are going to prove that every ordinal $\alpha$ with $\varepsilon_{0}>\alpha \geq \omega^{\omega}$ satisfies a natural zero one law in the following sense. For $\alpha<\varepsilon_{0}$ let $N \alpha$ be the number of occurences of $\omega$ in the Cantor normal form of $\alpha$. $(N \alpha$ is then the number of edges in the unordered tree which can canonically be associated with $\alpha$.) We prove that for any $\alpha$ with $\omega^{\omega} \leq$ $\alpha<\varepsilon_{0}$ and any sentence $\varphi$ in the language of linear orders the limit $\delta_{\varphi}(\alpha)=\lim _{n \rightarrow \infty} \frac{\#\{\beta<\alpha:(\beta, \in) \mid=\varphi \wedge N \beta=n\}}{\#\{\beta<\alpha: N \beta=n\}}$ exists and that $\delta_{\varphi}(\alpha) \in\{0,1\}$. We further show that for any such sentence $\varphi$ the limit $\delta_{\varphi}\left(\varepsilon_{0}\right)$ exists although this limit is in general in between 0 and 1 . We also investigate corresponding asymptotic densities for ordinals below $\omega^{\omega}$.
\end{abstract}

\section{Introduction}

This paper concerns logical limit laws for infinite ordinals. It is based on methods and techniques from the theory of logical limit laws for classes of finite structures and some ingredients from the theory of linear orders. We heavily use the machinery (which to a large extent goes back to pioneering work of Compton) developed in the book by Burris [2].

In 2001 the first author (after having read [2] and having recognized that ordinals below $\varepsilon_{0}$ form a natural additive number system) discussed the possibility of limit laws for ordinals with Compton at an AOFA-workshop in Tatihoo and Compton suggested among other things to contact the second author because of his results on limit laws for trees. This led to a fruitful interaction over the years. Tragically the second author passed away at the age of 58 unexpectedly in december 2011 and the first author feels responsible to make publically available the beautiful results which so far have been achieved (partially funded by DFG and the John Templeton Foundation).

Technically this article is based on a mixture of results from [4] (which when compared with the results from this article have preliminary nature) and [7]. Also some basic techniques from [2], [3] (and implicitly [1]) are used.

More elaborate results of the authors (concerning larger ordinal segments and more general languages, like the second order monadic ones) on limit laws for ordinals are sketched at the end and will hopefully be treated at later occasions. 


\section{Some basic results}

For two linear orders $A$ and $B$ let $G_{n}(A, B)$ be the following two person game, also well known as Ehrenfeucht Fraïsse game, between, lets say, Bob and Alice. A play consists of an ordered sequence of $n$ repetitions of the following: Bob chooses an element of $A$ or $B$ and Alice chooses an element of the other. The element of $A$ selected at the $t$-th turn is denoted $a_{t}$ and the element of $B$ selected at the $t$-th turn is denoted $b_{t}$. We say that Alice has won the game if for each $s, t \leq n$ the assertion $a_{s}<a_{t}(\bmod A)$ is equivalent with $b_{s}<b_{t}(\bmod B)$. Otherwise we say that Bob has won. As usual we define what a winning strategy for Alice is and we say $A \sim_{n} B$ iff Alice has a winning strategy for $G_{n}(A, B)$. (We took this standard exposition from page 99 in Rosenstein's classical text book [3] on linear orders.) Let $\mathcal{L}$ be the usual first order language of linear orders. Then $\alpha \sim_{n} \beta$ yields that $\alpha$ and $\beta$ model exactly the same $\mathcal{L}$-sentences $\varphi$ of quantifier rank not exceeding $n$.

Lemma 1 Fix a natural number $n \geq 1$. Let $\left(a_{i}\right)_{i \leq n}$ and $\left(a_{i}^{\prime}\right)_{i \leq n}$ be sequences of natural numbers such that for all $i \leq n:\left(a_{i} \geq 2^{n} \Longleftrightarrow a_{i}^{\prime} \geq 2^{n}\right)$ and $\left(a_{i}<2^{n} \Rightarrow a_{i}=a_{i}^{\prime}\right)$.

Then we have

$$
\omega^{n} \cdot a_{n}+\cdots+\omega^{0} \cdot a_{0} \sim_{n} \omega^{n} \cdot a_{n}^{\prime}+\cdots+\omega^{0} \cdot a_{0}^{\prime} .
$$

Moreover for such sequences $\left(a_{i}\right)_{i \leq n}$ and $\left(a_{i}^{\prime}\right)_{i \leq n}$ and for any pair of non zero ordinals $\alpha$ and $\beta$ we have

$$
\omega^{n+1} \cdot \alpha+\omega^{n} \cdot a_{n}+\cdots+\omega^{0} \cdot a_{0} \sim_{n} \omega^{n+1} \cdot \beta+\omega^{n} \cdot a_{n}^{\prime}+\cdots+\omega^{0} \cdot a_{0}^{\prime} .
$$

Proof. We have only to show assertion (1) (but we still need assertion (2) for applying the induction hypothesis). For, we have $\omega^{n+1} \cdot \alpha \sim_{2 n+2} \omega^{n+1}$ and $\omega^{n+1} \cdot \beta \sim_{2 n+2} \omega^{n+1}$ by assertion (1) of Theorem 6.18 in [3]. Assertion (1) above and closure of $\sim_{n}$ onder sums (i.e. assertion (1) of Lemma 6.5 of [3]) together with downward conservativity (cf., e.g. Lemma 6.4 of [3]) then yield $\omega^{n+1} \cdot \alpha+\omega^{n} \cdot a_{n}+\cdots+\omega^{0} \cdot a_{0} \sim_{n} \omega^{n+1} \cdot \beta+\omega^{n} \cdot a_{n}^{\prime}+\cdots+\omega^{0} \cdot a_{0}^{\prime}$. For proving assertion (1) we proceed by induction on $n$. The induction starts with $n=1$ for which the assertion holds. Now consider

$$
\gamma=\omega^{n} \cdot a_{n}+\cdots+\omega^{0} \cdot a_{0}
$$

and

$$
\delta=\omega^{n} \cdot a_{n}^{\prime}+\cdots+\omega^{0} \cdot a_{0}^{\prime} .
$$

We are going to apply Theorem 6.6 in [3] i.e. we verify the Fraïsse conditions for games on linear orderings. So we have to show that for every splitting of $\gamma(\delta)$ into an initial and final segment there is a splitting of $\delta(\gamma)$ into corresponding initial and final segments for which the second player wins the corresponding games on initial and final segments separately with $n-1$ moves. 
Assume without loss of generality that Player one picks $\xi<\gamma$. (In case that he picks $\xi<\delta$ the argument is symmetrical.) Assume that

$$
\xi=\omega^{n} \cdot a_{n}+\cdots+\omega^{i+1} \cdot a_{i+1}+\omega^{i} \cdot b_{i}+\cdots+\omega^{0} \cdot b_{0}
$$

and $b_{i}<a_{i}$.

Assume first that $a_{i} \geq 2^{n}$.

In this case also $a_{i}^{\prime} \geq 2^{n}$. If $b_{i}<2^{n-1}$ let $b_{i}^{\prime}:=b_{i}$. If If $b_{i} \geq 2^{n-1}$ and $a_{i}-b_{i}<2^{n-1}$ let $b_{i}^{\prime}:=a_{i}^{\prime}-\left(a_{i}-b_{i}\right)$ ensuring $b_{i}^{\prime} \geq 2^{n-1}$. If $b_{i} \geq 2^{n-1}$ and $a_{i}-b_{i} \geq 2^{n-1}$ then let $b_{i}^{\prime}:=2^{n-1}$. Let

$$
\xi^{\prime}:=\omega^{n} \cdot a_{n}^{\prime}+\cdots+\omega^{i+1} \cdot a_{i+1}^{\prime}+\omega^{i} \cdot b_{i}^{\prime}+\cdots+\omega^{0} \cdot b_{0}
$$

and let Player two play $\xi^{\prime}$. By induction hypothesis applied to (2) and assertion (1) of Lemma 6.5 in [3] we obtain $\xi \sim_{n-1} \xi^{\prime}$. So the initial segments determined by $\xi$ and $\xi^{\prime}$ are $\sim_{n}$ equivalent and it remains that the final segments in $\gamma$ and $\delta$ are also $\sim_{n}$ equivalent. To prove this let

$$
X:=\{\eta: \xi<\eta<\gamma\}
$$

and

$$
Y:=\left\{\eta: \xi^{\prime}<\eta<\delta\right\}
$$

For a set $X$ of ordinals let as usual otype $(X)$ denote its order type, i.e. the ordinal which is order isomorphic to it. Then

$$
\operatorname{otype}(X)=\omega^{i} \cdot\left(a_{i}-b_{i}\right)+\omega^{i-1} \cdot a_{i-1}+\cdots+\omega^{0} \cdot a_{0} .
$$

If $b_{i}<2^{n-1}$ then

$$
\operatorname{otype}(Y)=\omega^{i} \cdot\left(a_{i}^{\prime}-b_{i}\right)+\omega^{i-1} \cdot a_{i-1}^{\prime}+\cdots+\omega^{0} \cdot a_{0}^{\prime}
$$

where $a_{i}-b_{i} \geq 2^{n-1}$ since $a_{i}^{\prime} \geq 2^{n}$.

If $b_{i} \geq 2^{n-1}$ and $a_{i}-b_{i}<2^{n-1}$ then

$$
\begin{aligned}
\operatorname{otype}(Y) & =\omega^{i} \cdot\left(a_{i}^{\prime}-\left(a_{i}^{\prime}-\left(a_{i}-b_{i}\right)\right)+\omega^{i-1} \cdot a_{i-1}^{\prime}+\cdots+\omega^{0} \cdot a_{0}^{\prime}\right. \\
& =\omega^{i} \cdot\left(a_{i}-b_{i}\right)+\omega^{i-1} \cdot a_{i-1}^{\prime}+\cdots+\omega^{0} \cdot a_{0}^{\prime} .
\end{aligned}
$$

If $b_{i} \geq 2^{n-1}$ and $a_{i}-b_{i} \geq 2^{n-1}$ then

$$
\operatorname{otype}(Y)=\omega^{i} \cdot\left(a_{i}^{\prime}-2^{n-1}\right)+\omega^{i-1} \cdot a_{i-1}^{\prime}+\cdots+\omega^{0} \cdot a_{0}^{\prime}
$$

where $a_{i}^{\prime}-2^{n-1} \geq 2^{n-1}$ since $a_{i}^{\prime} \geq 2^{n}$. By induction hypothesis applied to (2) and closure of $\sim_{n-1}$ under sum (assertion (1) of Lemma 6.5 in [3]) we obtain $X \sim_{n-1} Y$.

Assume for the second case that $a_{i}<2^{n}$. In this case by assumption on the $a_{i}$ and $a_{i}^{\prime}$ we have necessarily $a_{i}^{\prime}=a_{i}$. Let

$$
\xi^{\prime}:=\omega^{n} \cdot a_{n}^{\prime}+\cdots+\omega^{i+1} \cdot a_{i+1}^{\prime}+\omega^{i} \cdot b_{i}+\cdots+\omega^{0} \cdot b_{0}
$$


be the response of Player two. To apply Theorem 6.6 of [3] we split as in the first case $\gamma$ and $\delta$ into initial and final segments. By induction hypothesis applied to (2) and assertion (1) of Lemma 6.5 in [3] we obtain $\xi \sim_{n-1} \xi^{\prime}$. As before let

$$
X:=\{\eta: \xi<\eta<\gamma\}
$$

and

$$
\begin{gathered}
Y:=\left\{\eta: \xi^{\prime}<\eta<\delta\right\} . \\
\operatorname{otype}(X)=\omega^{i} \cdot\left(a_{i}-b_{i}\right)+\omega^{i-1} \cdot a_{i-1}+\cdots+\omega^{0} \cdot a_{0}
\end{gathered}
$$

and

$$
\operatorname{otype}(Y)=\omega^{i} \cdot\left(a_{i}^{\prime}-b_{i}\right)+\omega^{i-1} \cdot a_{i-1}^{\prime}+\cdots+\omega^{0} \cdot a_{0}^{\prime} .
$$

By induction hypothesis applied to $(2)$ and closure of $\sim_{n-1}$ under sum we obtain $X \sim_{n-1} Y$. The assertion now follows from Theorem 6.6 of [3].

Let us define for each $\alpha<\varepsilon_{0}$ the norm of $\alpha, N \alpha$, as follows by recursion on $\alpha . N 0:=0$ and $N \alpha:=n+N \alpha_{1}+\cdots+N \alpha_{n}$ if $\alpha$ has Cantor normal form $\alpha=\omega^{\alpha_{1}}+\cdots+\omega^{\alpha_{n}}$. Then $N \alpha$ is the number of occurrences of $\omega$ in the Cantor normal form of $\alpha$. If we associate with $\alpha$ an unordered tree in the canonical way (see the proof of Theorem 2) then $N \alpha$ is the number of edges in the tree representation of $\alpha$ and so $N$ is in fact a canonical norm function on the ordinals below $\varepsilon_{0}$. For a given $\alpha<\varepsilon_{0}$ and $n<\omega$ there will only be finitely many $\xi<\alpha$ with $N \xi=n$. For a finite set $M$ we denote its cardinality by $\# M$. The ordinal $\varepsilon_{0}$ is as usual the least ordinal $\xi$ such that $\xi=\omega^{\xi}$.

For $\alpha<\varepsilon_{0}$ we therefore may then define

$$
c_{\alpha}(n)=\#\{\xi<\alpha: N \xi=n\}
$$

and for an $\mathcal{L}$-sentence $\varphi$ we may further define

$$
c_{\alpha}^{\varphi}(n)=\#\{\xi<\alpha: \xi \models \varphi \wedge N \xi=n\} .
$$

We further define

$$
\delta_{\varphi}(\alpha):=\lim _{n \rightarrow \infty} \frac{c_{\alpha}^{\varphi}(n)}{c_{\alpha}(n)}
$$

if this limit exists. We say that $\alpha$ satisfies an $\mathcal{L}$-limit law if $\delta_{\varphi}(\alpha)$ exists for all $\varphi$ and we say that $\alpha$ satisfies an $\mathcal{L}$ zero one law if $\delta_{\varphi}(\alpha)$ exists for all $\varphi$ and if $\delta_{\varphi}(\alpha)$ is either 0 or 1 . In the sequel we write $\alpha \models \varphi$ as an abbreviation for $(\alpha, \in) \models \varphi$.

Theorem 1 Let $\varepsilon_{0}>\alpha \geq \omega$. Then $\omega^{\alpha}$ satisfies an $\mathcal{L}$ zero one law.

Proof. Let $\varphi$ be a sentence of the language of linear orders. Assume that $n$ is the quantifier rank of $\varphi$. Let $I$ be the set $\left\{\{0\},\{1\}, \ldots,\left\{2^{n}-1\right\},\left\{m: m \geq 2^{n}\right\}\right\}$. Let $A_{0}, \ldots, A_{n}$ be a sequence of elements of $I$. Let

$P_{\alpha}\left(A_{0}, \ldots, A_{n}\right):=\left\{\omega^{n+1} \cdot \beta+\omega^{n} \cdot a_{n}+\cdots+\omega^{0} \cdot a_{0}<\omega^{\alpha}: \beta>0 \wedge(\forall i \leq n)\left[a_{i} \in A_{i}\right]\right\}$ 
and

$$
Q_{\alpha}\left(A_{0}, \ldots, A_{n}\right):=\left\{\omega^{n} \cdot a_{n}+\cdots+\omega^{0} \cdot a_{0}<\omega^{\alpha}:(\forall i \leq n)\left[a_{i} \in A_{i}\right]\right\} .
$$

Then the union of $P_{\alpha}$ 's and $Q_{\alpha}$ 's taken over all (finitely many) sequences of elements in $I$ gives the set of all ordinals below $\omega^{\alpha}$. By the Lemma 1 we have $\gamma \sim_{n}$ $\delta$ for any pair of elements of each of the sets $P_{\alpha}\left(A_{0}, \ldots, A_{n}\right)$ and $Q_{\alpha}\left(A_{0}, \ldots, A_{n}\right)$. The finite collection of the $P_{\alpha}\left(A_{0}, \ldots, A_{n}\right)$ and $Q_{\alpha}\left(A_{0}, \ldots, A_{n}\right)$ yields an effective (finite) description of the finitely many equivalence classes for $\sim_{n}$. Then

$$
\left\{\pi<\omega^{\alpha}: \pi \models \varphi\right\}=\bigcup_{A_{0}, \ldots, A_{n} \in I:\left(\exists i A_{i} \neq\{\emptyset\}\right) \wedge \exists \xi \in P_{\alpha}\left(A_{0}, \ldots, A_{n}\right): \xi \models \varphi} \bigcup_{A_{0}, \ldots, A_{n} \in I:\left(\exists i A_{i} \neq\{\emptyset\}\right) \wedge \exists \xi \in Q_{\alpha}\left(A_{0}, \ldots, A_{n}\right): \xi \models \varphi} P_{\alpha}\left(A_{0}, \ldots, A_{n}\right) \cup
$$

and we only have to show that the asymptotic density of each $P_{\alpha}\left(A_{0}, \ldots, A_{n}\right)$ and $Q_{\alpha}\left(A_{0}, \ldots, A_{n}\right)$ is either 0 or 1 . Of course the value 1 can only be distributed once. Consider a set $P_{\alpha}\left(A_{0}, \ldots, A_{n}\right)$ where some $A_{i}$ is a singleton containing exactly $a_{i}$. Let $\mathcal{P}_{n+1}:=\left\{\omega^{\eta}: \alpha>\eta \geq n+1\right\}$. Let $\mathcal{P}_{i}:=\left\{\omega^{i}\right\}$ for $i \leq n$. Then $P_{\alpha}\left(A_{0}, \ldots, A_{n}\right)$ is a partition set in the sense of Burris [2] and can be written as

$$
P\left(A_{0}, \ldots, A_{n}\right)=(\geq 0) \mathcal{P}_{n+1}+\cdots+a_{i} \cdot \mathcal{P}_{i} \cdots .
$$

This means that elements of $P\left(A_{0}, \ldots, A_{n}\right)$ can be written as sums of arbitrary many members of $\mathcal{P}_{n+1}$ and $a_{i}$ members of each $\mathcal{P}_{i}$ for $i \leq n$. We have that the local count function $c_{\omega^{\alpha}}(n)$ for $\omega^{\alpha}$ is in $R T_{1}$ by [4] and according to Compton's Theorem 4.1 in [2] we obtain that the asymptotic density of $P_{\alpha}\left(A_{0}, \ldots, A_{n}\right)$ is zero since $a_{i}$ is a small index (following the terminology of Definition 3.23 in [2]. If all $A_{i}$ are non singleton elements then Theorem 4.1 in [2] shows that the asymptotic density of $P_{\alpha}\left(A_{0}, \ldots, A_{n}\right)$ is one. Finally since $Q\left(A_{0}, \ldots, A_{n}\right)$ can be written following Definition 3.25 of [2] as $0 \cdot \mathcal{P}_{n+1}+\cdots$ Theorem 4.1 in [2] yields that the asymptotic density of $Q\left(A_{0}, \ldots, A_{n}\right)$ is zero since 0 is a small index. $\square$

Theorem 2 For any $\mathcal{L}$ sentence $\varphi$ the limit $\delta_{\varphi}\left(\varepsilon_{0}\right)$ exists.

Proof. The proof starts as the proof of Theorem 1 and continues with Woods' tree theorem.

Let $\varphi$ be a sentence of the language of linear orders. Assume that $n$ is the quantifier rank of $\varphi$. Let $I$ be the set $\left\{\{0\},\{1\}, \ldots,\left\{2^{n}-1\right\},\left\{m: m \geq 2^{n}\right\}\right\}$. Let $A_{0}, \ldots, A_{n}$ be a sequence of elements of $I$. Let

$P\left(A_{0}, \ldots, A_{n}\right):=\left\{\omega^{n+1} \cdot \beta+\omega^{n} \cdot a_{n}+\cdots+\omega^{0} \cdot a_{0}<\varepsilon_{0}: \beta>0 \wedge(\forall i \leq n)\left[a_{i} \in A_{i}\right]\right\}$

and

$$
Q\left(A_{0}, \ldots, A_{n}\right):=\left\{\omega^{n} \cdot a_{n}+\cdots+\omega^{0} \cdot a_{0}<\varepsilon_{0}:(\forall i \leq n)\left[a_{i} \in A_{i}\right]\right\} .
$$

Then the union of $P$ 's and $Q$ 's taken over all (finitely many) sequences of elements in $I$ gives the set of all ordinals below $\varepsilon_{0}$. By Lemma 1 we have $\gamma \sim_{n} \delta$ for 
any pair of elements of fixed set $P$ or a fixed set $Q$. Thus we have an effective description of the finitely many equivalence classes for $\sim_{n}$. Then

$$
\left\{\pi<\varepsilon_{0}: \pi \models \varphi\right\}=\bigcup_{A_{0}, \ldots, A_{n} \in I:\left(\exists i A_{i} \neq\{\emptyset\}\right) \wedge \exists \xi \in P\left(A_{0}, \ldots, A_{n}\right): \xi \models \varphi} P_{\alpha}\left(A_{0}, \ldots, A_{n}\right) \cup
$$

and we only have to show we only have to show that the asymptotic density of each $P\left(A_{0}, \ldots, A_{n}\right)$ and $Q\left(A_{0}, \ldots, A_{n}\right)$ exists. Now consider a fixed $P\left(A_{0}, \ldots, A_{n}\right)$ (or $Q\left(A_{0}, \ldots, A_{n}\right)$ ). To each ordinal $\alpha$ less than $\varepsilon_{0}$ we may associate canonically a finite rooted non planar tree $T(\alpha)$ as follows. (Non planar refers in contrast to planar to the property that there is no canonical ordering assumed for the immediate subtrees of a given tree.) To 0 we associate the tree consisting of a root. If $\alpha$ has a normal form $\omega^{\alpha_{1}}+\cdots+\omega^{\alpha_{n}}$ then we may assume that we have associated trees $T\left(\alpha_{i}\right)$ to the ordinals $\alpha_{i}$ for $1 \leq i \leq n$. Now let $T(\alpha)$ be the tree consisting of a root and immediate subtrees $T\left(\alpha_{1}\right), \ldots, T\left(\alpha_{n}\right)$. Then $|T(\alpha)|$, the number of nodes of $T(\alpha)$, is $1+N(\alpha)$. By the finitary character of the description of $P\left(A_{0}, \ldots, A_{n}\right)$ we may find a sentence $\psi$ in the language of trees such that $P\left(A_{0}, \ldots, A_{n}\right)=\left\{\alpha<\varepsilon_{0}: T(\alpha) \models \psi\right\}$. This follows from Lemma 1 and the fact that one can describe trees representing ordinals of the form $\omega^{k}$ by a first order formula in the language of trees. Theorem 1.1 of the second author from [7] yields that for any monadic second order property $\psi$ the limiting distribution probability of the fraction of unlabelled rooted trees with $n$ vertices and which satisfy $\psi$ exists. Therefore

$$
\lim _{n \rightarrow \infty} \frac{\#\{T: T=\psi \wedge|T|=n+1\}}{\#\{T:|T|=n+1\}}
$$

exists. But this limit is equal to

$$
\lim _{n \rightarrow \infty} \frac{\#\left\{\alpha<\varepsilon_{0}: T(\alpha) \models \psi \wedge N \alpha=n\right\}}{\#\left\{\alpha<\varepsilon_{0}: N \alpha=n\right\}}
$$

which is the asymptotic density of $P\left(A_{0}, \ldots, A_{n}\right)$.

It is clear that we cannot expect a zero one law for $\epsilon_{0}$. Being a successor is a first order property which has in this case a limiting distribution probability strictly inbetween 0 and 1 as shown in [4].

\section{Refinements}

We now investigate limit laws for not necessarily additive principal ordinals below $\varepsilon_{0}$. 
Lemma 2 Let $\alpha=\omega^{\beta} \cdot m+\gamma$ where $m>0$ and $\gamma<\omega^{\beta}<\varepsilon_{0}$. Let $\varphi$ be an $\mathcal{L}$-sentence. Assume that

$$
\delta_{\varphi}\left(\omega^{\beta} \cdot m\right)=\lim _{n \rightarrow \infty} \frac{c_{\omega^{\beta} \cdot m}^{\varphi}(n)}{c_{\omega^{\beta} \cdot m}(n)}
$$

exists. Then

$$
\lim _{n \rightarrow \infty} \frac{c_{\alpha}^{\varphi}(n)}{c_{\alpha}(n)}=\delta_{\varphi}\left(\omega^{\beta} \cdot m\right) .
$$

Proof. This Lemma is an easy corollary of the proof of Lemma 3.1 in [4].

$$
\begin{aligned}
\frac{c_{\alpha}^{\varphi}(n)}{c_{\alpha}(n)} & \geq \frac{c_{\omega^{\beta} \cdot m}^{\varphi}(n)}{c_{\alpha}(n)} \\
& =\frac{c_{\omega^{\beta} \cdot m}(n)}{c_{\alpha}(n)} \cdot \frac{c_{\omega^{\beta} \cdot m}^{\varphi}(n)}{c_{\omega^{\beta} \cdot m}(n)} \rightarrow_{n \rightarrow \infty} 1 \cdot \delta_{\varphi}\left(\omega^{\beta} \cdot m\right)
\end{aligned}
$$

by equation (2) in the proof of Lemma 3.1 of [4]. Moreover

$$
\begin{aligned}
\frac{c_{\alpha}^{\varphi}(n)}{c_{\alpha}(n)} \leq & \frac{c_{\omega^{\beta} \cdot m}^{\varphi}(n)}{c_{\omega^{\beta} \cdot m}(n)}+\frac{\#\left\{\xi: \omega^{\beta} \cdot m \leq \xi<\alpha \wedge N \xi=n\right\}}{c_{\omega^{\beta} \cdot m}(n)} \\
& \rightarrow_{n \rightarrow \infty} \delta_{\varphi}\left(\omega^{\beta} \cdot m\right)+0
\end{aligned}
$$

by equation (2) in the proof of Lemma 3.1 of of [4].

Lemma 3 Assume that $\alpha<\omega^{\omega}$ where $\alpha=\omega^{k} \cdot m_{k}+\cdots+\omega^{0} \cdot m_{0}$ with $m_{k}>0$. Then for each $\ell \in\left\{0, \ldots, m_{k}\right\}$ there is a sentence $\varphi \in \mathcal{L}$ such that

$$
\lim _{n \rightarrow \infty} \frac{c_{\alpha}^{\varphi}(n)}{c_{\alpha}(n)}=\frac{\ell}{m_{k}} .
$$

Proof. Let $\varphi$ describe that there exist exactly $\left(m_{k}-\ell\right) k$-limit points. (Recall that 1-limit points are just limit points, i.e. ordinals of the form $\dot{\omega(1+\beta)}$ and recall that $k$-limit points are limits of $(k-1)$-limit points. So $k$-limit points have the form $\omega^{k}(1+\beta)$. Obviously being a $k$-limit point is first order definable property of an ordinal under consideration.)

We are going to prove that

$$
\lim _{n \rightarrow \infty} \frac{c_{\omega^{k} \cdot m_{k}}^{\varphi}(n)}{c_{\omega^{k} \cdot m_{k}}(n)}=\frac{\ell}{m_{k}} .
$$

This yields the claim by Lemma 2 . We first see that

$$
\begin{aligned}
&\left\{\delta<\omega^{k} \cdot m_{k}: \delta=\varphi\right.\wedge N \delta=n\}= \\
&\left\{\delta<\omega^{k} \cdot m_{k}: \delta \geq \omega^{k} \cdot\left(m_{k}-\ell\right)\right.\wedge N \delta=n\}= \\
&\left\{\xi<\omega^{k} \cdot l: N \xi=n-N\left(\omega^{k} \cdot\left(m_{k}-\ell\right)\right)\right\}
\end{aligned}
$$

Thus $c_{\omega^{k} \cdot m_{k}}^{\varphi}(n)=c_{\omega^{k} \cdot \ell}\left(n-N\left(\omega^{k} \cdot\left(m_{k}-\ell\right)\right)\right) \sim c_{\omega^{k}}(n) \cdot \ell$ as shown in the last line of the proof of Lemma 3.1 of [4] of [4]. Moreover $c_{\omega^{k} \cdot m_{k}}(n) \sim m \cdot c_{\omega^{k}}(n)$ and the assertion follows. 
Lemma 4 If $\gamma \geq \omega>m>0$ then

$$
\lim _{n \rightarrow \infty} \frac{c_{\omega \gamma \cdot m}^{\varphi}(n)}{c_{\omega \gamma \cdot m}(n)}=\lim _{n \rightarrow \infty} \frac{c_{\omega^{\gamma}}^{\varphi}(n)}{c_{\omega \gamma}(n)} \in\{0,1\} .
$$

Proof. Fix $\varphi$. Assume that the quantifier rank of $\varphi$ does not exceed the natural number $r$. For $2 \leq i \leq m$ let

$$
M_{i}^{1}(n):=\left\{\delta<\omega^{\gamma} \cdot i: \delta \models \varphi \wedge N \delta=n \wedge \delta \geq \omega^{\gamma} \cdot(i-1)+\omega^{r+1}\right\}
$$

and

$M_{i}^{2}(n):=\left\{\delta<\omega^{\gamma} \cdot i: \delta \models \varphi \wedge N \delta=n \wedge \omega^{\gamma} \cdot(i-1) \leq \delta<\omega^{\gamma} \cdot(i-1)+\omega^{r+1}\right\}$

Then

$$
\begin{array}{r}
c_{\omega^{\gamma} \cdot m}^{\varphi}(n) \geq \\
\#\left\{\delta<\omega^{\gamma} \cdot m: \delta \models \varphi \wedge N \delta=n \wedge \delta \geq \omega^{r}\right\}+ \\
\# M_{2}^{1}(n)+\# M_{2}^{2}(n)+\ldots+\# M_{m}^{1}(n)+\# M_{m}^{2}(n) .
\end{array}
$$

By Assertion (1) of Theorem 6.18 in [3] we know that $\omega^{r} \sim_{2 \cdot r} \omega^{r} \cdot \beta$ for all $\beta>0$. If $\delta=\omega^{\gamma} \cdot i+\xi$ where $\xi<\omega^{\gamma}$ and $i>0$ then, since $\gamma \geq \omega, \delta=\omega^{r} \cdot \omega^{\gamma} \cdot i+\xi$ and we have the equivalence: $\delta \models \varphi \Longleftrightarrow \omega^{r}+\xi \models \varphi$. Thus $\delta \mapsto \omega^{r}+\xi$ gives a projection into $\omega^{\gamma}$ which preserves the validity (invalidity) of $\varphi$. Assume first that $\lim _{n \rightarrow \infty} \frac{c_{\omega \gamma}^{\varphi}(n)}{c_{\omega} \gamma(n)}=1$. The remaining case $\lim _{n \rightarrow \infty} \frac{c_{\omega \gamma}^{\varphi}(n)}{c_{\omega \gamma} \gamma(n)}=0$ can be treated similarly. Now we have

$$
\# M_{i}^{1}(n)=\#\left\{\xi<\omega^{\gamma}: \xi \models \varphi \wedge N \xi=n-(N \gamma+1) \cdot i\right\}
$$

hence

$$
\frac{\# M_{i}^{1}(n)}{m \cdot c_{\omega \gamma}(n)}
$$

converges to $\frac{1}{m}$. We have

$$
\# M_{i}^{2}(n)=\#\left\{\xi<\omega^{r+1}: \xi \models \varphi \wedge N \xi=n-(N \gamma+1) \cdot i+r+1\right\}
$$

hence

$$
\frac{\# M_{i}^{2}(n)}{m \cdot c_{\omega \gamma}(n)}
$$

converges to 0 . We see that $\liminf _{n \rightarrow \infty} \frac{c_{\omega \gamma \cdot m}^{\varphi}(n)}{c_{\omega \gamma \cdot m}(n)} \geq 1$ and the assertion follows.

Corollary 1 If $\varepsilon_{0}>\gamma \geq \omega^{\omega}$ then $\gamma$ satisfies an $\mathcal{L}$ zero one law. 
Theorem 3 Assume that $\alpha<\omega^{\omega}$ where $\alpha=\omega^{k} \cdot m_{k}+\cdots+\omega^{0} \cdot m_{0}$ with $m_{k}>0$. Then for each sentence $\varphi \in \mathcal{L}$ there is an $\ell \in\left\{0, \ldots, m_{k}\right\}$ such that

$$
\lim _{n \rightarrow \infty} \frac{c_{\alpha}^{\varphi}(n)}{c_{\alpha}(n)}=\frac{\ell}{m_{k}} .
$$

Proof. By Lemma 2 we may assume without loss of generality that $\alpha=\omega^{k} \cdot m$. Let $\varphi$ be given and assume that the rank of $\varphi$ is $r$. Let $\mathcal{P}_{i}:=\left\{\omega^{i}\right\}$ for $i \leq k$. Then $\{\delta<\alpha: \delta \models \varphi\}$ can be written as a disjoint union of partition sets (in the sense of Definition 3.25 of [2]) in the form $\bigcup_{A_{0}, \ldots, A_{k}} A_{k} \mathcal{P}_{k}+\cdots+A_{0} \mathcal{P}_{0}$ where $A_{i}$ is an index in $\left\{0,1, \ldots, 2^{r}-1,\left(\geq 2^{r}\right)\right\}$. Now $A_{k}$ has to be finite by the choice of $\alpha$. If one $A_{i}$ is finite for some $i<k$ then $A_{k-1} \mathcal{P}_{k-1}+\ldots+A_{0} \mathcal{P}_{0}$ is a partition set in $\omega^{k}$ such that by Theorem 4.1 of [2] we have

$$
\lim _{n \rightarrow \infty} \# \frac{\left\{\delta \in A_{k-1} \mathcal{P}_{k-1}+\ldots+A_{0} \mathcal{P}_{0}: N \delta=n\right\}}{c_{\omega^{k}}(n)}=0 .
$$

Thus we may concentrate on $A_{k} \mathcal{P}_{k}+\left(\geq 2^{r}\right) \mathcal{P}_{k-1}+\cdots+\left(\geq 2^{r}\right) \mathcal{P}_{0}$. This set has the same asymptotic density as $A_{k} \mathcal{P}_{k}$ and this set has asymptotic density in the desired set according to the shape of $A_{k}$. Moreover the resulting finite sums of these densities are also in the desired set of values.

Remarks: One referee of this paper suggested to report about possible extensions of the results of this paper. We give a short list but proofs will be reported elsewhere.

1. Let $\left(p_{i}\right)_{i \geq 1}$ be an enumeration of the prime numbers and for $\alpha<\varepsilon_{0}$ with Cantor normal form $\alpha=\omega^{\beta}+\gamma$ let $G \alpha:=p_{G \beta} \cdot G \gamma$ where $G 0:=1$. (This Gödel numbering shows up in Schütte's proof theory book and is also known as Matula-coding.) Let

$$
\Delta_{\varphi}(\alpha):=\lim _{n \rightarrow \infty} \frac{\#\{\beta<\alpha: \beta \models \varphi \wedge G \beta \leq n\}}{\#\{\beta<\alpha: G \beta \leq n\}} .
$$

Then $\Delta_{\varphi}(\alpha) \in\{0,1\}$ for $\omega^{\omega} \leq \alpha<\varepsilon_{0}$ and $\varphi \in \mathcal{L}$. This follows by adapting the proofs of this paper to the new situation since we are working with multiplicative numbers systems (in the sense of [2]) and we know from [4] that we have for $\alpha<\varepsilon_{0}$ that

$$
n \mapsto \#\{\beta<\alpha: G \beta \leq n\} \in R V_{0} .
$$

2. Let $\left(p_{i}\right)_{i \geq 1}$ again be the enumeration of the prime numbers and for $\alpha<\varepsilon_{0}$ with Cantor normal form $\alpha=\omega^{\alpha_{1}}+\cdots+\omega^{\alpha_{n}}$ let $G^{\prime} \alpha:=p_{1}^{G^{\prime} \alpha_{1}} \cdot \ldots \cdot p_{n}^{G^{\prime} \alpha_{n}}$ where $G^{\prime} 0:=1$. (Such a Gödel numbering shows up in Gödel's work).) Let

$$
\Delta_{\varphi}^{\prime}(\alpha):=\lim _{n \rightarrow \infty} \frac{\#\left\{\beta<\alpha: \beta \models \varphi \wedge G^{\prime} \beta \leq n\right\}}{\#\left\{\beta<\alpha: G^{\prime} \beta \leq n\right\}} .
$$

Then we conjecture that $\Delta_{\varphi}^{\prime}(\alpha) \in\{0,1\}$ for $\omega^{\omega} \leq \alpha<\varepsilon_{0}$ and $\varphi \in \mathcal{L}$. We expect that this follows by adapting the proofs of this paper to the new 
situation. To carry this out it seems reasonable to investigate whether we have for $\alpha<\varepsilon_{0}$ that

$$
n \mapsto \#\left\{\beta<\alpha: G^{\prime} \beta \leq n\right\} \in R V_{0} .
$$

A remaining problem is that with respect to $G^{\prime}$ we do not have a multiplicative number system in the sense of Burris [2].

3. Let us now come back to the additive situation which is based on the norm function $N$. If $\varphi$ is in the monadic second order language of linear orders then a limit law (but in general no zero one law) can be proved for all $\alpha$ with $\omega^{\omega} \leq \alpha \leq \varepsilon_{0}$. A proof for this result has been obtained by applying as new ingredient Shelah's 'additive colouring' technique.

4. If $\varphi$ is in the weak monadic second order language of linear orders with + then a limit law can be proved for $\varepsilon_{0}$ but no algorithm can separate formulas having limiting probability 0 from formulas having limiting probability 1 .

5. If $\varphi$ is in the weak monadic second order language of linear orders with + and $\cdot$ then a limit law can be proved for the thinned out domain of structures $A=\left\{\omega^{\alpha}: \alpha<\varepsilon_{0}\right\}$.

6. One referee of this paper asked whether limit laws are affected by the choice of notation. Using a formula by Lagrange 1775 (cf. Lemma 3.3 in [5]) it can easily be shown that an $\mathcal{L}$ zero one law breaks down for many ordinals between $\omega^{\omega}$ and $\varepsilon_{0}$ when they are represented by the lexicographic path order over a signature with a binary function symbol and a constant (cf., e.g. the definition of $<$ in [5] p.6). But the expectation is that for any system of ordinal notations published in the literature at least limit laws will hold with respect to $\mathcal{L}$ and canonically extended norm functions. For more expressive languages one would expect Cesaro limit laws to hold in many situations covered by 'natural wellorderings' (cf., e.g., [6]).

\section{References}

1. J.P. Bell, S.N. Burris: Asymptotics for logical limit laws: when the growth of the components is in an RT class. Trans. Amer. Math. Soc. 355 (2003), no. 9, 37773794

2. S.N. Burris: Number Theoretic Density and Logical Limit Laws. Mathematical Surveys and Monographs 86. American Mathematical Society 2001.

3. J. Rosenstein. Linear Orderings. Academic Press 1982.

4. A. Weiermann: A zero one law characterization of $\varepsilon_{0}$. Mathematics and Computer Science II. Proceedings of the Colloquium on Algorithms, Trees, Combinatorics and Probabilities. Birkhäuser (2002), B. Chauvin, P. Flajolet, D. Gardy and A. Mokkadem (ed.), 527-539.

5. A. Weiermann: Phase transition thresholds for some Friedman-style independence results. Mathematical Logic Quarterly 53 (1), (2007), 4-18.

6. A. Weiermann: Analytic combinatorics, proof-theoretic ordinals, and phase transitions for independence results Annals of Pure and Applied Logic 136 (1-2) (2005), 189-218.

7. A.R. Woods: Coloring rules for finite trees, and probabilities of monadic second order sentences. Random Structures Algorithms, 10 (4), (1997) 453-485. 